7. Pfitzmann R, Benscheidt B, Langrehr JM, Schumacher $G$, Neuhaus $R$, Neuhaus $P$. Trends and experiences in liver retransplantation over 15 years. Liver Transplantation 2007; 13(2):248-257.

8. Kim WR, Lake JR, Smith JM, et al. OPTN/SRTR 2016 annual data report: liver. Am J Transplant 2018;18(18 Suppl 1):172-253.

9. Mortality and retransplantation. European Liver Transplant Registry. Available at http://www.eltr.org/Mortality-and-retransplantation.html

10. Allen AM, Kim WR, Xiong $H$, et al. Survival of recipients of livers from donation after circulatory death who are relisted and undergo retransplant for graft failure. Am J Transplant 2014;14:1120-1128.

11. Kalisvaart M, de Haan JE, Polak WG, Metselaar HJ, Wijnhoven BPL, IJzermans JNM, et al. Comparison of postoperative outcomes between donation after circulatory death and donation after brain death liver transplantation using the comprehensive complication index. Ann Surg 2017:266:772-8.

12. Matton AP, Porte RJ. Opportunities for scientific expansion of the deceased donor pool. Liver Transp/ 2014;20:S5

\section{P047 NUTRITION IN DECOMPENSATED LIVER DISEASE: A REGIONAL TRAINEE-LED AUDIT INVESTIGATING MALNUTRITION AND THE IMPACT OF NUTRITIONAL INTERVENTIONS FOLLOWING ADMISSION WITH DECOMPENSATED CHRONIC LIVER DISEASE}

\begin{abstract}
'James Morgan*, ${ }^{2}$ Lauren O'Flynn, ${ }^{2}$ Amar Srinivasa, ${ }^{2}$ Shahd Ahmed, ${ }^{2}$ Gohar Jalayeri Nia, ${ }^{1}$ Sara Mahgoub, ${ }^{2}$ Chemilie Ngonadi, ${ }^{2}$ Michael McFarlane, ${ }^{2}$ Nicola Burch, ${ }^{2}$ Victoria Gordon. ${ }^{1}$ University Hospital Birrmingham, UK; ${ }^{2}$ University Hospital Coventry and Warwickshire, Coventry, UK
\end{abstract}

\subsection{6/gutjnl-2021-BASL.56}

Introduction Malnutrition results in prolonged inpatient stay, high readmission rates and poor clinical outcome. ${ }^{1-3}$ Malnutrition in cirrhosis is a poor prognostic factor. ${ }^{4}$ Assessing nutritional status is problematic as traditional methods of assessment are inaccurate. ${ }^{5}$ Decompensated cirrhosis (DC) patients often require nutritional support but optimal timing of intervention and outcomes are unclear. ${ }^{6}{ }^{7}$ A regional audit assessing the prevalence of malnutrition in DC, nutritional management, impact on length of stay, readmission and mortality was conducted.

Method All adults admitted with DC from 1-30 November 2019 to four acute $\mathrm{xxxx}$ hospital Trusts were retrospectively identified from coding. Malnutrition was assessed by MUST and Royal Free Hospital-Nutritional Prioritisation Tool (RFHNPT) screening tools. ${ }^{8}$

Results 47 patients were identified. Patient demographics, liver disease aetiology, baseline biochemical, disease severity, nutritional assessment and mortality were collected (table 1). Prevalence of malnutrition was $76.6 \%$ (CI 62-87.6\%) which was independent of age or aetiology of liver disease but was associated with disease severity. There were higher rates of screening on gastroenterology wards $(p=0.012)$ compared to other wards. Nutritional supplementation was prescribed in 27 $(60 \%)$ patients, $78 \%$ of which was oral supplementation. Median length of hospitalisation was 9 (2-62) days. This was longer in those prescribed nutritional supplements (median 11:7 days). Those prescribed supplements were more likely to have more severe liver disease (MELD 20:15; $\mathrm{p}=0.006$ ). Readmission rates at 1 month were similar in those prescribed nutritional supplements than those not (35\% vs 36\%). Mortality was higher in malnourished patients $(p=0.03)$ and in the group of patients prescribed nutritional supplements versus not at 1,3 and 6 months.

Conclusions Malnutrition is widespread in DC being present in $76.6 \%$ of this cohort as compared to $30 \%$ in hospitalised patients. Malnutrition was independently associated with early readmission and mortality. The RFH-NPT was significantly
Abstract P047 Table 1 Patient demographics, liver disease aetiology, baseline biochemical data, disease severity, nutritional assessment and patient mortality $(n=47)$

\begin{tabular}{|c|c|}
\hline \multicolumn{2}{|l|}{ Parameter } \\
\hline Male:female $n(\%)$ & $26: 21(55: 45)$ \\
\hline Age in years (median (range)) & $62(16-86)$ \\
\hline \multicolumn{2}{|l|}{ Liver disease aetiology $\mathbf{n}(\%)$} \\
\hline Alcohol related liver disease (ARLD) & $22(47)$ \\
\hline Non-alcoholic fatty liver disease (NAFLD) & $12(26)$ \\
\hline Viral & $8(17)$ \\
\hline Autoimmune & $3(6)$ \\
\hline Other & $2(4)$ \\
\hline \multicolumn{2}{|l|}{ Disease severity: } \\
\hline Child Pugh n (\%) & $\begin{array}{l}A-4(8), B-22(47), C-21 \\
(45)\end{array}$ \\
\hline MELD (median (range)) & $20(6-32)$ \\
\hline \multicolumn{2}{|l|}{ Patient management } \\
\hline Patients admitted to Gastroenterology ward n (\%) & $36(77)$ \\
\hline \multicolumn{2}{|l|}{ Nutritional assessment } \\
\hline Malnourished by MUST n (\%) & $26(55)$ \\
\hline Malnourished by RFH-NPA n (\%) & $36(77)$ \\
\hline Malnourished $\mathrm{n}(\%)$ by Child Pugh & $\begin{array}{l}A-2(50), B-16(72), C-18 \\
(86)\end{array}$ \\
\hline Patients screened for malnutrition (Gastro ward) n (\%) & $30(83)$ \\
\hline $\begin{array}{l}\text { Patients screened for malnutrition (Non-Gastro ward) } n \\
(\%)\end{array}$ & $5(45)$ \\
\hline Time to nutritional/dietetic assessment (median (range)) & $4(1-14)$ \\
\hline Patients receiving dietician review (Gastro ward) $\mathrm{n}(\%)$ & $19(52)$ \\
\hline $\begin{array}{l}\text { Patients receiving dietician review (Non-Gastro ward) n } \\
(\%)\end{array}$ & $4(45)$ \\
\hline \multicolumn{2}{|l|}{ Patient mortality } \\
\hline $\begin{array}{l}\text { No nutritional supplements prescribed at } 1,3 \text { and } 6 \\
\text { months } n(\%)\end{array}$ & $3(18), 4(24), 6(35)$ \\
\hline $\begin{array}{l}\text { Nutritional supplements prescribed at } 1,3 \text { and } 6 \text { months } \\
\mathrm{n}(\%)\end{array}$ & $10(33), 14(47), 14(47)$ \\
\hline
\end{tabular}

more sensitive for detecting malnutrition in cirrhosis compared to MUST. Though screening was better on Gastroenterology wards, it is of concern that this was incomplete for many patients nor was a dietician review accomplished in all malnourished patients. Our results indicate that initial in-patient nutritional interventions do not appear to impact on early readmission or early mortality. A larger multi-centre audit would be required to evaluate the impact of specific nutritional interventions on longer-term outcomes. We propose early management of malnutrition at all possible clinical interactions with specialty dietician involvement. Both primary and secondary care guidance could be adapted to achieve this.

\section{REFERENCES}

1. Kyle UG, Genton L, Pichard C. Hospital length of stay and nutritional status. Curr Opin Clin Nutr Metab Care 2005;8(4):397-402.

2. Lim SL, Ong KCB, Chan YH, et al. Malnutrition and its impact on cost of hospitalization, length of stay, readmission and 3-year mortality. Clin Nutr 2012;31 (3):345-350.

3. Charlton $\mathrm{K}$, Nichols $\mathrm{C}$, Bowden $\mathrm{S}$, et al. Poor nutritional status of older subacute patients predicts clinical outcomes and mortality at 18 months of follow-up. Eur $J$ Clin Nutr 2012;66(11):1224.

4. McFarlane $M$, Hammond $C$, Roper $T$, et al. Comparing assessment tools for detecting undernutrition in patients with liver cirrhosis. Clin Nutr ESPEN 2018:23:156-161

5. Cheung K, Lee SS, Raman M. Prevalence and mechanisms of malnutrition in patients with advanced liver disease, and nutrition management strategies. Clin Gastroenterol Hepatol 2012;10(2):117-125. 
6. European Association for the Study of the Liver. EASL Clinical Practice Guidelines on nutrition in chronic liver disease. J Hepatol 2019;70(1):172-193.

7. Plauth $M$, Bernal W, Dasarathy $S$, et al. ESPEN guideline on clinical nutrition in liver disease. Clin Nutr 2019;38(2):485-521.

8. Arora S, Mattina C, Catherine M, et al. PMO-040 the development and validation of a nutritional prioritising tool for use in patients with chronic liver disease. Gut 2012;61:A90

\section{P048 GEOGRAPHIC VARIABILITY IN RATES OF INTENSIVE CARE UNIT ADMISSION IN PATIENTS WITH CHRONIC LIVER DISEASE AND CRITICAL COVID-19: INTERNATIONAL REGISTRY DATA}

${ }^{1}$ Thomas Marjot* ${ }^{2}$ Andrew Moon, ${ }^{3}$ Matthew Armstrong, ${ }^{4}$ Ignacio García-Juárez, ${ }^{5}$ Anand Kulkarni, ${ }^{6}$ Steven Masson, ${ }^{7}$ Nneka Ufere, ${ }^{8}$ David Wong, COVID-Hep \& SECURELiver contributors ${ }^{1,9}$, ${ }^{2}$ Luke Baldelli, ${ }^{2}$ Alfred Barritt, ${ }^{1}$ Eleanor Barnes, ${ }^{9} \mathrm{G}$ wilym Webb. ${ }^{1}$ Oxford Liver Unit, Translational Gastroenterology Unit, Oxford University Hospitals NHS Foundation Trust, University of Oxford, Oxford, UK; ${ }^{2}$ Division of Gastroenterology and Hepatology, University of North Carolina, Chapel Hill, North Carolina, USA; ${ }^{3}$ Liver Unit, Queen Elizabeth Hospital Birmingham, Birmingham, UK; ${ }^{4}$ Department of Gastroenterology, Instituto Nacional de Ciencias Médicas y Nutrición Salvador Zubirán, Mexico City, Mexico; ${ }^{5}$ Dept. of Hepatology and Liver Transplantation, Asian Institute of Gastroenterology Hospitals, Gachibowli, Hyderabad, India; 'Liver Transplant Unit, Freeman Hospital, The Newcastle upon Tyne Hospitals NHS Foundation Trust, Newcastle upon Tyne, UK; ' Liver Center, Gastrointestinal Division, Massachusetts General Hospital, Harvard Medical School, Boston, Massachusetts, USA; ${ }^{8}$ Toronto Centre for Liver Disease, University Health Network, University of Toronto, Canada; ${ }^{9}$ Cambridge Liver Unit, Addenbrooke's Hospital, Cambridge University Hospitals, Cambridge, UK

10.1136/gutjnl-2021-BASL.57

Background and Aims The COVID-19 pandemic has provided a unique opportunity to evaluate global intensive care unit (ICU) admission practices for a common indication. Patients with chronic liver disease (CLD) and cirrhosis may also have limited or variable access to ICU. We aimed to describe international ICU admission rates and outcomes in patients with CLD and critical COVID-19.

Methods Data were combined from two international registries (SECURE-Liver and COVID-Hep) for patients with CLD and COVID-19 which was deemed severe enough to require ICU by the reporting clinician. Rates of ICU admission or decline, and respective outcomes were compared by country.

Results Between 25th March 2020 and 3rd February 2021, 319 patients with CLD and COVID-19 from 27 countries were judged to require ICU. The proportion of patients ultimately accepted to ICU varied according to country (figure
1A), although mortality following ICU admission was similar by country (figure 1B). Factors associated with being declined ICU admission included advancing age, cirrhosis, alcohol related liver disease, and UK origin. To explore national differences further, we compared cases from the USA and UK, the two greatest contributing countries. Rates of ICU admission differed significantly between the USA and UK [77/79 $(95 \%)$ vs. $22 / 77$ (29\%); p<0.001]. However, there were no differences in mortality in those admitted to ICU $(42 / 75$ [56\%] vs. $10 / 22$ [45\%]; $\mathrm{p}=0.468$; figure $1 \mathrm{~B})$, or after receiving invasive ventilation $(29 / 59$ [49\%] vs. 9/17 [53\%]; $\mathrm{p}=1.000)$. There were also no differences in age, sex, Charlson Comorbidity Index, or baseline liver disease severity between countries, both in those requiring and admitted to ICU. This included comparable rates of cirrhosis (53/79 [67\%] vs. $56 / 77[72 \%] ; p=0.723)$. Only four USA patients were declined ICU admission of whom $2(50 \%)$ died, whereas 55 UK patients were declined ICU admission of whom 51 (93\%) died. In both USA and UK cohorts, the reason for not admitting patients to ICU was due to this being deemed inappropriate by the responsible clinician, except for one case in both countries where no ICU bed was available. Notably, information relating to patient wishes, long-term outcomes in survivors, and granular detail regarding organ support requirements were not available.

Conclusion Patients with CLD and critical COVID-19 were over 3-times more likely to be admitted to ICU in the USA than the UK despite having similar baseline characteristics. However, the rates of mortality following ICU admission were comparable between the two countries. The differing thresholds for escalation to ICU but similar post admission outcomes warrants further discussion.

\section{P049 DECOMPENSATED LIVER DISEASE: A QUALITY IMPROVEMENT PROJECT}

Rebecca Osborne*, Geeta Prasad. Wythenshawe Hospital, Manchester, UK

\subsection{6/gutjnl-2021-BASL.58}

Decompensated liver disease is considered a medical emergency with an associated high morbidity and mortality (10$20 \%)^{1}$. It is a common presentation to hospital with multi-

B
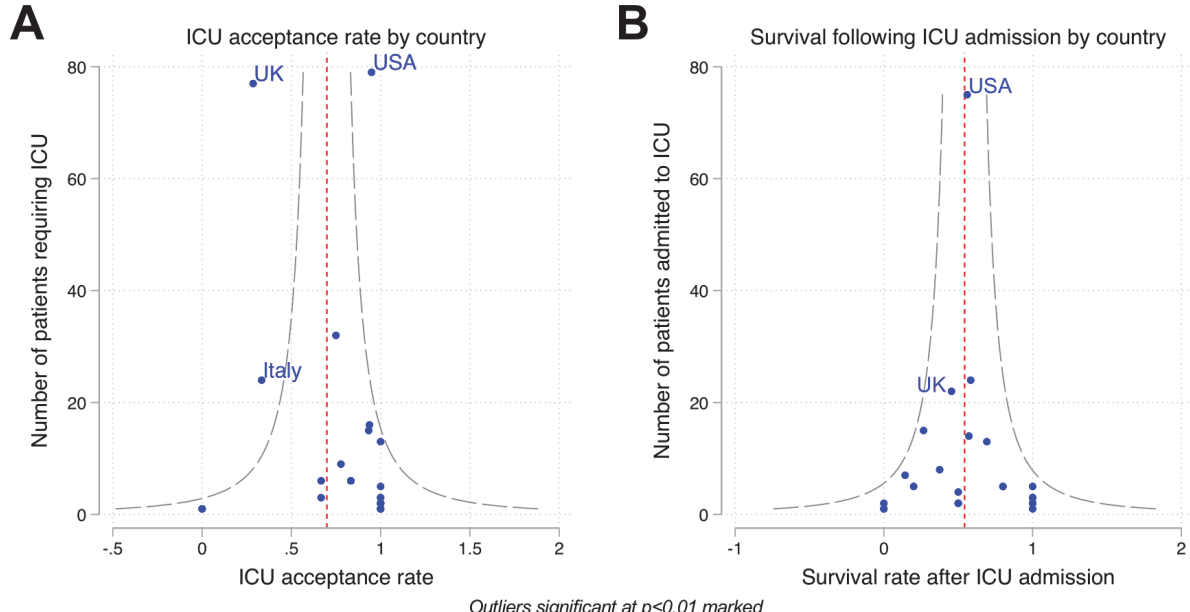